\title{
Recall mode and recency in immediate serial recall: Computer users beware!
}

\author{
CATHERINE G. PENNEY and PENNY ANN BLACKWOOD \\ Memorial University of Newfoundland, St. John's, Newfoundland, Canada
}

\begin{abstract}
Two experiments were carried out in which subjects were tested on immediate serial recall of digit lists. In both experiments, some subjects wrote their responses on paper, and other subjects entered their responses by means of a computer keyboard. In both experiments, keyboard entry of responses resulted in lower recall of items from the recency part of the serial-position curve, and in the second experiment, the difference between the two response modes was greater when lists had been presented visually rather than auditorily. The auditory suffix effect was not diminished by keyboard entry of responses. Investigators of recency effects in serial recall are cautioned about using keyboard entry of responses.
\end{abstract}

The use of computers has become widespread in experimental psychology, notably in the collection of data in studies of human memory. The benefits are obvious and do not need to be described here. In changing from the "old-fashioned" procedure of having subjects write down their responses, to the more efficient method of having them enter responses on a computer keyboard, we noted an interesting effect on performance in immediate serial-recall tasks. Keyboard entry reduced recall, especially of items from the recency part of the serial-position curve. In the present article, we report the results of two experiments in which keyboard and handwritten response modalities were compared.

\section{EXPERIMENT 1}

The first experiment was performed to investigate the suffix effect in the Hebb repetition task. Each subject had 60 trials in which immediate serial recall of 10-digit lists was required. Unbeknownst to the subject, six of the lists were repeated eight times each throughout the series. Although recall of repeating lists also showed the same effects, it is the data from the 12 nonrepeating lists that are presented here. (The results from the repeating lists will be reported elsewhere.)

\section{Method}

Subjects. A total of 96 subjects was tested, with 12 males and 12 females in each combination of the suffix and no-suffix conditions with keyboard and written response modes. All subjects answered advertisements posted around the campus offering payment for their participation. Most subjects had previously participated in memory experiments, but none had experience in experiments on the suffix effect.

The research reported here was supported by Grant A9587 from the Natural Sciences and Engineering Research Council (NSERC) to the first author and an NSERC summer award to the second author. We would like to thank A. Kerry Butt for his assistance with computer programming. Correspondence may be addressed to Catherine G. Penney, Psychology Department, Memorial University of Newfoundland, St. John's, Newfoundland A1B 3X9 Canada.
Design and Procedure. Eighteen sequences of 10 digits were generated for each subject, each sequence consisting of a random permutation of the digits 0 through 9 . Twelve of these lists were presented only once, whereas the other 6 were presented eight times each for a total of 60 experimental lists per subject. The spacing between repetitions of a given list varied from four to seven intervening lists.

Presentation of stimuli was controlled by a Polymorphic System 8813 computer fitted with a Quintrex Articulator. Auditory presentation was used throughout. The articulator allows digital storage of analog input. Thus, the digits 0 through 9 and the word "now" were digitized, trimmed to $.5 \mathrm{sec}$, and stored on disk. The digit 0 was pronounced "oh," and 7 was pronounced insofar as possible as a one-syllable word. At presentation, the digits were converted back to analog and presented through a loudspeaker. The digits were presented at the rate of one every $.75 \mathrm{sec}$. In the suffix condition, the word "now" followed each list in rhythm. In the keyboard condition, the subjects entered their responses on the computer keyboard, pressing the "return" key after each number and entering " $x$ " when they could not recall a digit. In the written recall condition, the subjects wrote each digit on a supplied response sheet in left-to-right order, leaving a blank for a digit that could not be recalled. The importance of the left-to-right response order was emphasized by the experimenter, who watched carefully during practice trials to see that the instructions were followed. After recalling a sequence, the subject pressed any key to proceed to the next list. Two seconds later, the word "ready" appeared in the middle of the screen, followed, after another $2 \mathrm{sec}$, by the next list.

Subjects were tested individually. Each subject read an instruction sheet explaining the task and the recall requirements. The subjects then received 10 practice trials, after which any questions about procedure were answered and the 60 experimental trials began. The subjects covered their response sheets so that they could not look back at previously recalled lists. After the trials had concluded, the subjects were informed of the nature of the experiment and were paid.

\section{Results}

For the nonrepeated lists, the proportions of items recalled in the correct positions were subjected to an ANOVA with response modality and suffix as betweensubjects factors and serial position and block as withinsubject factors. The 12 nonrepeating lists presented to each subject were divided into two blocks of six trials each so that the effects of practice on the task could be analyzed.

The typical effects of suffix condition and serial position were found. The suffix reduced recall from $48.4 \%$ to $41.4 \%\left[F(1,92)=8.19, M S_{\mathrm{e}}=.288, p<.01\right]$. The 
Table 1

Percentage of Recall for Each Serial Position in Experiment 1

\begin{tabular}{cccccc}
\hline \multirow{2}{*}{$\begin{array}{c}\text { Serial } \\
\text { Position }\end{array}$} & \multicolumn{2}{c}{ No Suffix } & & \multicolumn{2}{c}{ Suffix } \\
\cline { 2 - 3 } \cline { 5 - 5 } \cline { 5 - 5 } & Keyboard & Written & & Keyboard & Written \\
\hline 1 & 89.6 & 87.4 & & 82.3 & 84.6 \\
2 & 68.8 & 70.9 & & 69.1 & 63.5 \\
3 & 58.7 & 57.2 & & 52.1 & 49.2 \\
4 & 43.4 & 44.9 & & 45.5 & 45.5 \\
5 & 33.7 & 35.5 & & 35.4 & 27.6 \\
6 & 29.2 & 26.1 & & 28.1 & 25.2 \\
7 & 21.5 & 23.0 & & 18.9 & 22.8 \\
8 & 20.1 & 27.7 & 13.5 & 27.4 \\
9 & 27.4 & 50.3 & & 11.5 & 37.7 \\
10 & 59.4 & 92.5 & 24.3 & 63.1 \\
\hline
\end{tabular}

suffix effect was localized to the end of the list, with the interaction between suffix condition and serial position reaching significance $\left[F(9,1311)=11.6, M S_{\mathrm{e}}=.040\right.$, $p<.001]$. The usual U-shaped serial-position curves were found $\left[F(9,207)=151, M S_{\mathrm{e}}=.060, p<.001\right]$, with large primary and recency effects. (See Table 1 for percentages recalled.) The main effect of block was significant $\left[F(1,23)=11.0, M S_{\mathrm{e}}=.088, p<.005\right]$, with recall levels of $39.6 \%$ and $43.6 \%$, respectively, in the first and second blocks for keyboard responding, and $45.6 \%$ and $50.6 \%$, respectively, for handwritten responding. Block interacted with serial position $[F(9,207)=$ $2.32, M S_{\mathrm{e}}=.029, p<.05$ ], with the greatest increases with practice occurring for Serial Positions 4, 6, and 10.

The effects of interest are the main effect of response modality $\left[F(1,92)=7.05, M S_{\mathrm{e}}=.288, p<.01\right]$ and the interaction between response modality and serial position $\left[F(9,1311)=21.7, M S_{\mathrm{e}}=.040, p<.001\right]$. Table 1 presents the relevant data. Response modality produced a very large effect on the terminal list item in both the suffix and the no-suffix conditions. For the penultimate item, the effect was smaller; for the item in Serial Position 8, the effect was smaller still; and for items in Serial Position 7 and earlier, the effect was negligible. No other interactions reached significance.

\section{EXPERIMENT 2}

Experiment 2 was carried out to examine modality and suffix effects in forward and backward recall, but only the data from forward-recall trials are presented here. (A comparison of forward and backward recall will be reported elsewhere.) Half of the subjects entered their responses on the computer keyboard; the remaining subjects wrote their responses.

\section{Method}

Subjects. Ten male and 8 female university students were tested on each response modality. Their ages ranged from 18 to 28 years. Subjects volunteered for the experiment by signing a list posted in the psychology department offering money for participating. Many subjects had taken part in previous memory experiments in the department.

Design and Procedure. Eight conditions forming a $2 \times 2 \times 2$ design were tested in a randomized order in each response modality. The variables were list modality (auditory or visual), suffix modality (also auditory or visual), and direction of recall (forward or backward). There were 10 lists in each of the eight conditions, making a total of $80 \mathrm{ex}$ perimental trials.

Eighty lists of nine digits were generated, consisting of random orderings of the digits 0 through 9 , with the tenth digit dropped. Presentation of lists and suffixes, and randomization of the conditions was done using a Polymorphic 8813 computer fitted with a Quintrex Articulator as in Experiment 1. The visual lists were presented on a Hitachi blackand-white monitor. The digits were presented, either auditorily or visually, by the computer at the rate of one item per $0.7 \mathrm{sec}$. In addition, a cue was presented at the end of each list indicating whether recall should proceed in forward or backward order. The cues were the words "front" and "back." The cue was presented either auditorily or visually, with modality of the cue being orthogonal to modality of the list items. Items were to be recalled in serial order, starting with either the first or the last item, as indicated by the cue. Half of the subjects recorded their responses by typing the digits on the computer keyboard and pressing the return key after each digit. The remaining subjects wrote their responses on a sheet of paper. They were instructed to write digits in a left-to-right order for both forward and backward recall, starting with the first item presented in the forward condition and the last item presented in the backward condition. All subjects were instructed to use the letter " $x$ " in place of any digit they could not recall.

At the beginning of the experiment, the subjects were given a typed sheet of instructions and 16 practice trials. Any questions about procedure were answered at this stage, and the 80 experimental trials followed. On a single trial, the word "ready" appeared on the screen $2 \mathrm{sec}$ prior to the first digit. The digits followed at a rate of $0.7 \mathrm{sec}$ per digit and then either the word "front" or "back" in rhythm with the digits. In the keyboard-recall condition, after the subject entered nine digits, the screen cleared and presentation of the next list began. After writing their responses, subjects in the written-recall condition pressed any key to initiate presentation of the next list. The subjects either entered or wrote an " $x$ " when they could not recall a particular digit. After the experiment was over, the subjects were informed of the purpose of the study, and any questions about the expected results or the theoretical interpretation were answered.

\section{Results}

An ANOVA was performed on data from forwardrecall trials only and included response modality as a between-subjects factor, and list modality, suffix modality, and serial position as within-subjects factors. The dependent variable was the number of digits recalled in the correct position for the 10 lists in each condition.

The main effect of list modality was not significant $[F(1,17)=1.29]$, but the interaction between list modality and serial position was significant $[F(8,136)=29.5$, $\left.M S_{\mathrm{e}}=2.15, p<.001\right]$. The typically superior recall of auditorily presented items from the end of the list was partly offset by the higher recall of visual items from the earlier serial positions. The effects of suffix mode $[F(1,17)$ $\left.=9.65, M S_{\mathrm{e}}=4.80, p<.01\right]$ and serial position $\left[F(8,136)=67.2, M S_{\mathrm{e}}=5.79, p<.001\right]$ were both significant, as was their interaction $\left[F(8,136)=14.2, M S_{\mathrm{e}}=\right.$ $1.22, p<.001]$. The three-way interaction between list modality, suffix modality, and serial position was also significant, $\left[F(8,136)=6.58, M S_{\mathrm{e}}=1.80, p<.001\right]$. The auditory suffix reduced recall of items from the end of the list, but the effect was found mainly for auditory lists.

The main effect of response mode did not reach statistical significance $[F(1,34)=2.69]$, but response mode interacted with both list modality $\left[F(1,595)=18.8, M S_{\mathrm{e}}=\right.$ $54.2, p<.001]$ and serial position $[F(8,595)=8.28$, $\left.M S_{\mathrm{e}}=54.2, p<.001\right]$. Figure 1 illustrates the results. For both auditory and visual lists, recall of recency items 

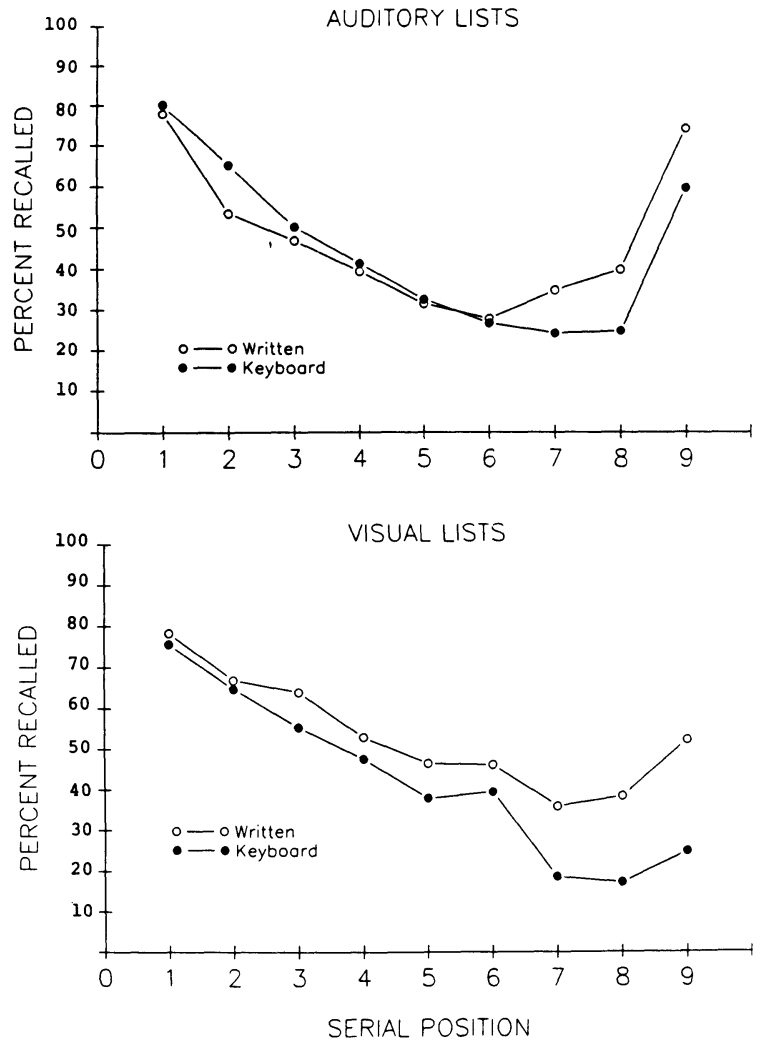

Figure 1. Serial-position curves for auditorily and visually presented lists with written and keyboard response modes.

was higher when subjects wrote their responses than when they entered them on the keyboard. The effect of response modality was larger for visual lists (means of $42.3 \%$ and $53.4 \%$ recall for keyboard and written response modes, respectively) than for auditory lists (means of $44.8 \%$ and $47.2 \%$ ). In fact, the subjects entering responses on the keyboard exhibited a minimal recency effect, which was restricted to the terminal list item.

\section{DISCUSSION}

The practical significance of the effects of using keyboard entry of responses is clear. The keyboard response mode reduced the recency effect substantially and, with visually presented lists, virtually eliminated the recency effect. The reduced size of the recency effect may decrease its sensitivity to some experimental manipulations. For this reason, experimenters investigating recency or suffix effects in immediate serial recall may wish to have subjects write down their responses rather than enter them directly on a computer keyboard.

The effect of response mode on recall of recency items also has some theoretical implications. Compare the effects of keyboard responding with oral recall. Oral recall selectively reduces recall of recency items, relative to written recall, when list presentation is auditory (Craik, 1969; Penney, 1979) or when list items are vocalized at input (Murray, 1966), but it appears that oral recall does not disrupt recall of visually presented items (Margrain, 1967; Murray, 1966). It therefore seems that the effect of oral recall is an example of modality-specific interference in that the sound of the subject's own voice seems to displace information from an echoic store.

In contrast, the keyboard response mode does not produce modalityspecific interference, but rather interference of a more general type. Had the keyboard response interfered selectively with an echoic memory representation, we would expect a smaller suffix effect and a smaller modality effect with the keyboard response. This did not occur. The interaction between response mode and suffix condition did not even approach significance for either experiment $(F \mathrm{~s}<1)$. In Experiment 2 , there was a significant interaction between list modality and response mode. For items from the last three serial positions, the difference in recall of auditory and visual items was larger with the keyboard response mode. Clearly, the interference produced by the keyboard response was not with an echoic memory representation.

One might also compare the effects of response modality to the effects of a redundant response prefix. (A prefix is usually a word spoken by the subject after the end of list presentation and before report of the memory items.) Whereas the keyboard response selectively reduced recall of recency items, the verbal response prefix has been shown to reduce recall of all items in the list (Crowder, 1967; Jahnke, Nowaczyk, \& Wozniak, 1976). Whatever the nature of the output interference produced by the verbal prefix, it does not appear to be the same as the interference produced by the keyboard response mode.

One possible interpretation of the results of the present experiments is that allocation of mental resources to the unpracticed keyboard response impairs retention of list items in a response buffer or rehearsal loop, or some other mechanism that is not modality-specific. The subjects who took part in these experiments were not skilled typists, and few had much experience with a computer keyboard. The keyboard response mode therefore required expenditure of more mental effort than did the more highly practiced written response. With the keyboard response, mental resources were thus diverted away from the maintenance of the list items, and the list items most susceptible to distraction are the items in the recency part of the list.

The conclusion that follows from the effects of oral and keyboard response is that there appear to be two components to the recency effect in auditorily presented lists. One component reflects the echoic memory, or auditory sensory store, and is susceptible to interference from a suffix or from the subject's oral recall. The second component reflects the operation of a mechanism that is not specific to the auditory modality but is subject to interference from the keyboard response. The recency effect in visual lists reflects a single component which is not modality-specific, this component probably being an output buffer or something similar.

\section{REFERENCES}

CraIK, F. I. M. (1969). Modality effects in short-term storage. Journal of Verbal Learning \& Verbal Behavior, 8, 658-664.

Crowder, R. G. (1967). Prefix effects in immediate memory. Canadian Journal of Psychology, 21, 450-461.

JAHNKe, J. C., NowaCzYK, R. H., \& WozNiak, W. (1976). Stimulus redundancy and immediate recall. Memory \& Cognition, 4, 357-360.

Margrain, S. (1967). Short-term memory as a function of input modality. Quarterly Journal of Experimental Psychology, 19, 109-114.

MURRAY, D. J. (1966). Vocalization at presentation and immediate recall, with varying recall methods. Quarterly Journal of Experimental Psychology, 18, 9-18.

Penney, C. G. (1979). Interactions of suffix effects with suffix delay and recall modality in serial recall. Journal of Experimental Psychology: Human Learning \& Memory, 5, 507-521.

(Manuscript received May 12, 1989.) 